\title{
Preparation and Properties of Starch-g-PLA with High Grafting Degree Catalyzed by Hydrochloric Acid
}

\author{
Xue Wang, Yingmo Hu*,Quan Liu, Mengcan Li, Chunyan Hou and Chao Zeng \\ School of Materials Science and Technology, China University of Geosciences, Beijing \\ *Ying mo Hu
}

Keywords: Starch; Lactic Acid, Graft Degree, Graft Copolymer, Degradable.

\begin{abstract}
Starch/lactic acid graft copolymer was attempted in situ grafting reaction of lactic grafting starch via the catalysis of hydrochloric acid. The graft copolymers of Starch and PLA had been synthesized and characterized by Fourier transform infrared spectroscopy (FT-IR), X-ray diffraction(XRD), and scanning electron microscopy(SEM). The synthetic condition were optimized that the weight ratios of starch with lactic acid were $1: 2$ when reacted for $3 \mathrm{hrs}$ at $90^{\circ} \mathrm{C}$ in $0.6 \mathrm{~mol} / \mathrm{L}$ hydrochloric acid solution. The starch-g-lactic acid copolymer can be finally obtained with graft degree of starch $65 \%$.
\end{abstract}

\section{Introduction}

Recently, the exploitation of starch based copolymer has applied in many field in reality. Such as the food packaging materials(Arun Ghosh, Tanushree Gupta, Jayanthi Swaminathan.2015[1]), the building materials(A.T.Le, A.Gacoin, A.Li,.2014 [2]) medical materials(Lei Ye,Yabin Zhang,Qiangsong Wang.2016.[3]) and any other fields. Compared with the plastic products from synthetic polymer, the copolymer of starch grafted with lactic acid made of renewable raw materials is friendly to the environment(Yingmo Hu, Qingling Wang, Mingru Tang.2013[4]). Therefore, Wang, Hu and Zhu(Wang, Q.L.,Hu,Y.M.,\&Zhu,J.H.2012 [5]) prepared starch-g-lactic directly by using the sodium hydroxide to catalyze with a grafting degree of 33.6\%. Yingmo Hu ,Mingru Tang(Yingmo Hu, Mingru Tang.2015 [6]) prepared starch-g-lactic acid directly via the reaction of starch with lactic acid catalyzed by ammonia water with grafting degree of $58.9 \%$. Although the procedure are convenient, the grafting degree is too low. By the way, the synthetic method of starch-g-lactic acid have to explore a new way to improve the grafting degree.

In this work, the starch grafting lactic copolymer was directly catalyzed by hydrochloric acid under gentle condition in order to produce the starch-g-lactic a higher grafting degree.

\section{Experimental}

\section{Materials:}

Potato starch was obtained from Beijing Gusong Economics \& Trade Co.Ltd. Hydrochloric acid (A.R), urea (A.R), ethanol (A.R) and acetone (A.R) were purchased from Beijing Chemical Works. Lactic acid (A.R) was obtained from Xilong Chemical Company.

\section{Preparation of Starch/lactic Acid Graft Copolymer(starch-g-PLA)}

Starch-g-PLA was prepared as follows: 5 graw sweet potato starch was dispersed in $30 \mathrm{~mL}$ urea solution. The mixture was heated to $80^{\circ} \mathrm{C}$ and stirred for about $2 \mathrm{hrs}$. then $\mathrm{HCl}$ and lactic acid were added to the flask to react at $90^{\circ} \mathrm{C}$ for $3 \mathrm{hrs}$ under vacuum condition. After system was cooled down to the room tempreture, ethyl alcohol is used to remove unreacted lactic acid monomer under vigorous stirring, then dried at $80^{\circ} \mathrm{C}$ to get the crude product. The dried product was further purified by Soxhlet extraction with acetone for $48 \mathrm{hrs}$ to remove the unreacted lactic acid monomer completely as well as the PLA homopolymer then dried at $80^{\circ} \mathrm{C}$. The final product with a grafting degree of $65 \%$ was obtained. 
The grafting degree was calculated as follows:

$$
\text { Grafting degree }=\frac{m 1-m 0}{m 0} * 100 \%
$$

Where $\mathrm{m} 0$ is the weight of dried raw starch and the $\mathrm{m} 1$ is the weight of the purified starch-g-PLA copolymer.

\section{Characterization}

Starch and its copolymer were characterized by IR (Perkin-Elmer 100) in the wavelength range from 4000 to $450 \mathrm{~cm}-1$, XRD (D/max-Ra diffractometer) with $\mathrm{CuK}$ a radiation $(\lambda=1.5406 \AA)$ operating at $40 \mathrm{kV}$ and $100 \mathrm{~mA}$ in the range of 5 50oof $2 \theta$ by steps of $0.02 \mathrm{o}$, and SEM (JEOL, JSM-7001F with resolution at $10 \mathrm{~m}, 5.0 \mathrm{KV}$ ), respectively.

\section{Results and Discussion}

\section{FT-IR Analysis}

The IR spectra of starch-g-PLA and sweet potato starch are shown in fig. 1 . The broad and strong absorption peak around $3500 \mathrm{~cm}^{-1}$ was attributed to the stretching vibration of -OH. The peak at 2930 $\mathrm{cm}^{-1}, 1648 \mathrm{~cm}^{-1}$ were assigned to the vibrational absorption of $\mathrm{C}-\mathrm{H}$ bond, intramolecular hydrogen bond in starch. The starch-g-PLA(Fig.1B) showed new strong peaks at $1737 \mathrm{~cm}^{-1}$ and $2982 \mathrm{~cm}^{-1}$, which were assigned to carbonyl of ester and $\mathrm{C}-\mathrm{H}$ bond in poly(lactic acid) chain, while the other absorption peaks all correspond to those of starch, which indicated that the starch-g-PLA was the product with the framework of starch grafted the lactic acid.

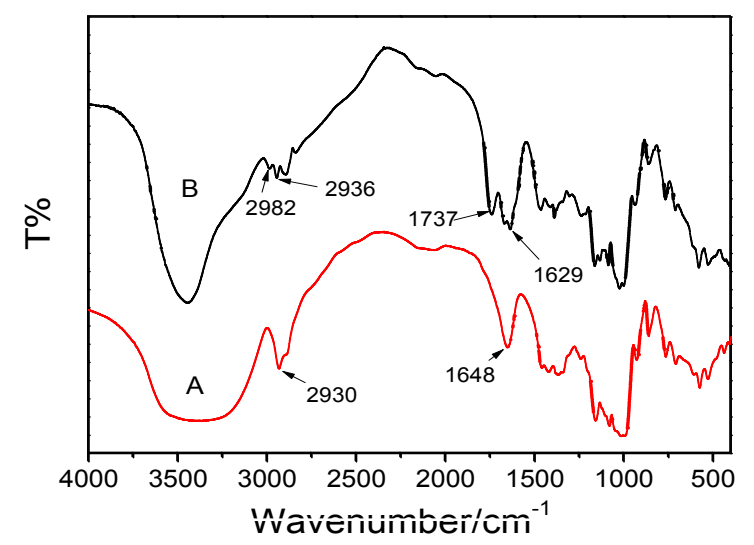

Fig 1. IR spectra of starch (A) and starch-g-lactic acid copolymer(B).

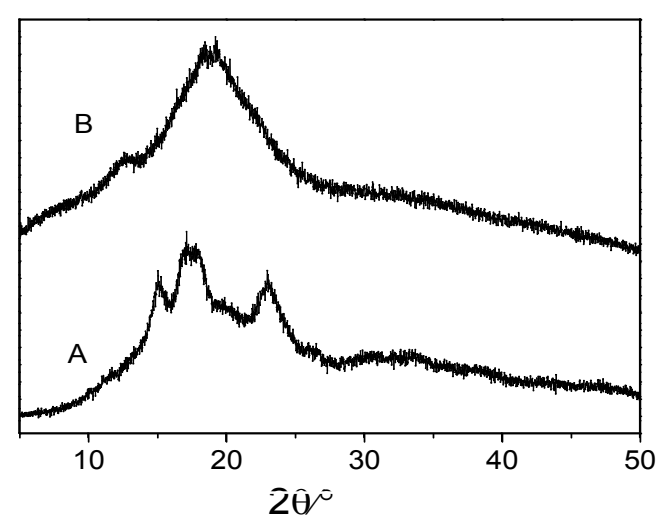

Fig 2. XRD of starch (A) and starch-g-lactic acid conolvmer(B).

\section{XRD Analysis}

The X-ray diffractogram of starch, starch-g-PLA are showed in fig.2, the starch(A) showed sharp peaks at $2 \theta=15^{\circ}, 17.3^{\circ}, 23^{\circ}$, demonstrating crystallization in starch, the starch-g-PLA(B) showed a dispersion band at around $2 \theta=19.2^{\circ}$ with the absence of the above three peaks, indicating that the starch-g-PLA is amorphous. 
The scanning electron microscopy images of starch and starch-g-PLA were shown in fig.3. The starch(fig.3A) showed the raw starch particles are round shaped, smooth space, dense structure without obvious edges. Comparing to the starch, the starch-g-PLA(fig.3B) changed its surface a lot with the coarse surface and inattentive particle liked structure. It indicated that starch-g-PLA is amorphous.

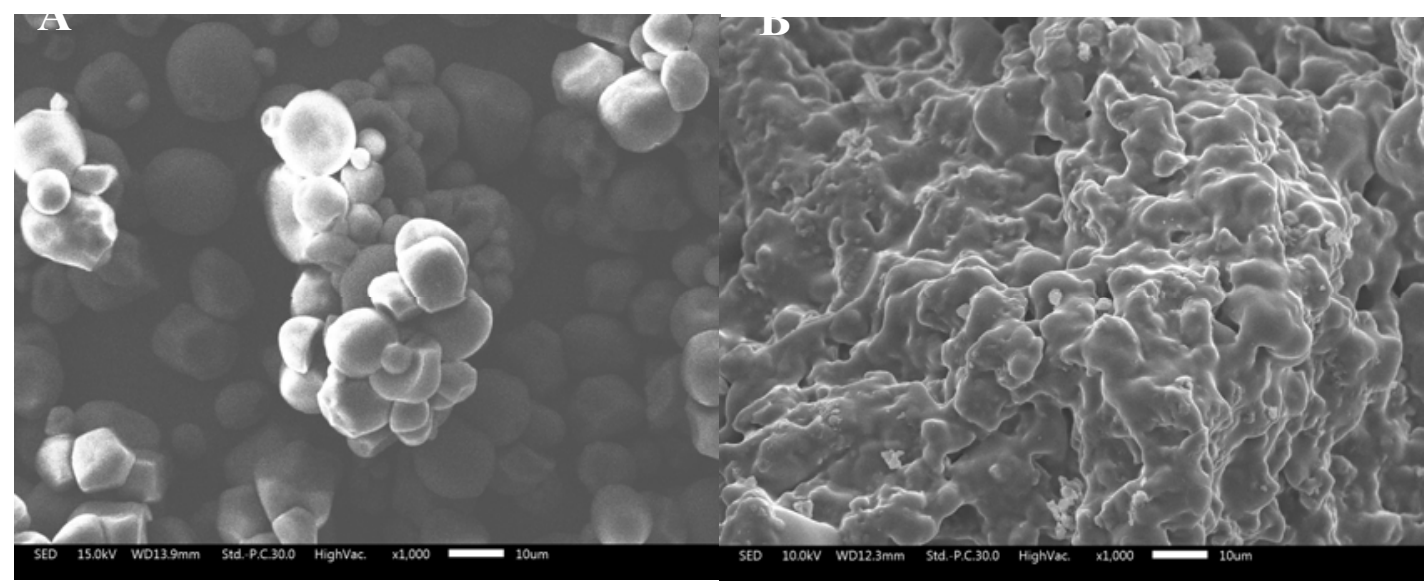

Fig 3. SEM images of starch (A) and starch-g-lactic acid copolymer(B).

\section{The Effects of NaOH Dosage and Weight Ratio of Starch to Lactic Acid on the Graft Degree of Starch}

Fig.4A showed that the effect of weight ratio of starch to lactic on the grafting copolymerization. With the addition of lactic, the grafting degree sharply increased till the ratio of starch to lactic acid reached 1:2. With the lactic concentration further increased, the grafting degree decreased on account of the excess lactic was leaded to homopolymerization. The effect of different concentration of $\mathrm{HCl}$ solution on the grafting degree of starch was shown in Fig.4B. $\mathrm{HCl}$ solution was regarded as a kind of green catalyst which is available and safe in operation. It could be found that the degree of starch increased with the dosage of $\mathrm{HCl}$ increased at initial situation. It decreased quickly with the $\mathrm{HCl}$ concentration increased after the $\mathrm{HCl}$ concentration up to $0.6 \mathrm{~mol} / \mathrm{L}$.

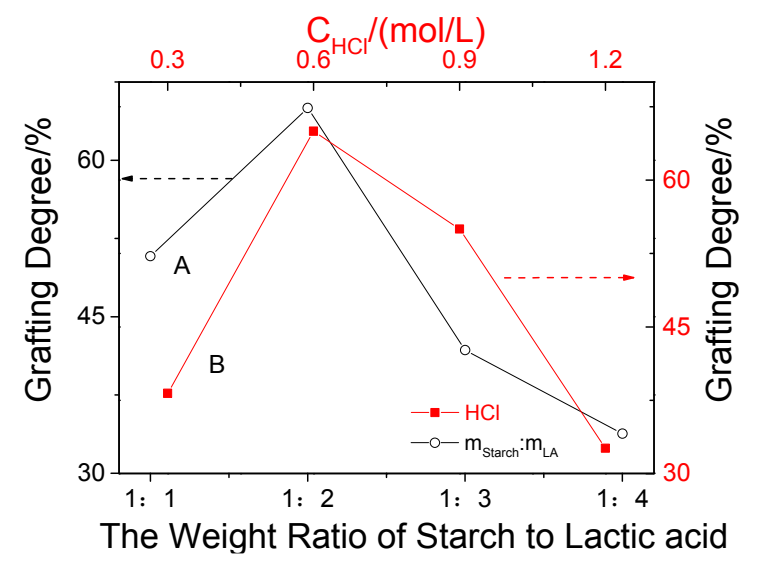

Fig 4. The effect of weight ratio of starch to lactic(A) and $\mathrm{NaOH}$ dosage(B) to graft degree of starch

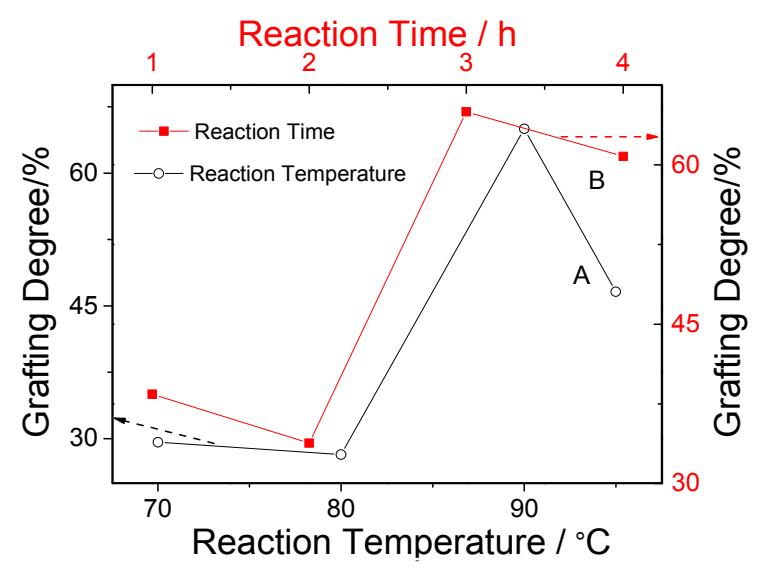

Fig 5. The effect of reaction temperature(A) and reaction time(B) to graft degree of starch 
Fig. 5 showed that the variation of reaction temperature and reaction time on grafting degree of starch. With the increasing reaction temperature, the grafting degree increased till the reaction temperature reached $90^{\circ} \mathrm{C}$ at initial stage and decreased when exceeding $90^{\circ} \mathrm{C}$, the tendency of curve of the reaction time was just like the reaction temperature, with the reaction time increased till 3 $\mathrm{hrs}$, the grafting degree increased, then with the react more time, the grafting degree dropped. It could be discribed that with increasing reaction temperature and prolonging reaction time below $90^{\circ} \mathrm{C}$ for $3 \mathrm{hrs}$, the easterification of the starch and lactic was more likely to react completely, while the hydrolysis would be accelerated when the reaction temperature get higher and the reaction time get longer.

\section{Conclusions}

The starch-g-lactic acid copolymer which catalyzed by hydrochloric acid was finally synthesized by one-step process in a mild condition. The structure of starch-g-lactic acid was characterized by FT-IR, XRD, SEM. The synthetic condition were optimized that the starch was activated at $80^{\circ} \mathrm{C}$ for $2 \mathrm{hrs}$, starch and lactic were added to reacted at $90^{\circ} \mathrm{C}$ for $3 \mathrm{hrs}$ with the ratio 1:2 in vacuum. Accordingly, the optimized grafting degree of the starch-g-lactic is $65 \%$. This work provided us a new way to the starch grafting procedure. The further study of its film procedure and mechanical performances is carrying though.

\section{Acknowledgement}

This work is supported by the Fundamental Research Funds for the Central Universities (2652016142).

\section{References}

[1] Arun Ghosh, Tanushree Gupta, Jayanthi Swaminathan, David Wright, Mechanical and anti-pathogenic characterization of starch-based materials, J. Polymer Testing. 43(2015)78-82.

[2] A.T.Le, A.Gacoin, A.Li, T.H.Mai, M.Rebay, Y.Delmas, Experimental investigation on the mechanical performance of starch-hemp composite materials, J. Construction and Building Materials. 61(2014)106-113.

[3] Lei Ye,Yabin Zhang,Qiangsong Wang,Xin Zhou,Boguang Yang,Feng Ji,Dianyu Dong,Lina Gao,Yuanlu Cui and Fanglian Yao, Physical cross-Linking starch-Based zwitterionic hydrogel exhibiting excellent biocompatibility,Protein Resistance, and Biodegradability, J. American Chemical Society. 8(2016)15710-15723

[4] Yingmo $\mathrm{Hu}$, Qingling Wang, Mingru Tang, Preparation and properties of Starch-g-PLA/poly(vinyl alcohol)composite film, J. Carbohydrate Polymers. 96(2013)384-388

[5] Wang, Q.L.,Hu,Y.M.,\&Zhu,J.H, Convenient synthetic method of starch/lactic acid graft copolymer catalyzed with sodium hydroxide, J. Bulletin of Material Science. 35(2012)415-418

[6] Yingmo Hu, Mingru Tang, Synthesis of starch-g lactic acid copolymer with high grafting degree catalyzed by ammonia water, J. Carbohydrate Polymers. 118(2015)79-82 